PROCEEDINGS OF THE

AMERICAN MATHEMATICAL SOCIETY

Volume 140, Number 10, October 2012, Pages 3593-3605

S 0002-9939(2012)11194-3

Article electronically published on February 23, 2012

\title{
COARSENING POLYHEDRAL COMPLEXES
}

\author{
NATHAN READING
}

(Communicated by Jim Haglund)

\begin{abstract}
Given a pure, full-dimensional, locally strongly connected polyhedral complex $\mathcal{C}$, we characterize, by a local codimension-2 condition, polyhedral complexes that coarsen $\mathcal{C}$. The proof of the characterization draws upon a general shortcut for showing that a collection of polyhedra is a polyhedral complex and upon a property of hyperplane arrangements which is equivalent, for Coxeter arrangements, to Tits' solution to the Word Problem. The motivating special case, the case where $\mathcal{C}$ is a complete fan, generalizes a result of Morton, Pachter, Shiu, Sturmfels, and Wienand that equates convex rank tests with semigraphoids. We also prove oriented matroid versions of our results, obtaining, as a byproduct, an oriented matroid version of Tietze's convexity theorem.
\end{abstract}

\section{Summary OF RESUlts}

The purpose of this paper is to characterize the polyhedral complexes $\mathcal{C}^{\prime}$ that coarsen a given polyhedral complex $\mathcal{C}$. A polyhedron is an intersection of finitely many closed half-spaces. A polyhedral complex is a finite, nonempty collection $\mathcal{C}$ of polyhedra such that (1) if $F \in \mathcal{C}$ and $G$ is a face of $F$, then $G \in \mathcal{C}$, and (2) if $F$ and $G$ are in $\mathcal{C}$, then $F \cap G$ is a face of $F$ and a face of $G$. The polyhedra in $\mathcal{C}$ are called the faces of $\mathcal{C}$. A fan is (the set of nonempty faces of) a polyhedral complex all of whose nonempty faces contain the origin. Details on polyhedra, polyhedral complexes and fans can be found, for example, in 10. We typically shorten "polyhedral complex" to "complex". The dimension of $\mathcal{C}$ is the maximum of the dimensions of its faces, and $\mathcal{C}$ is called pure if all of its maximal faces have the same dimension. The support $\operatorname{Supp}(\mathcal{C})$ of a collection $\mathcal{C}$ of polyhedra is the union of the polyhedra in the collection. A complex is complete if its support is the entire ambient space. A complex $\mathcal{C}^{\prime}$ coarsens a complex $\mathcal{C}$ if $\mathcal{C}^{\prime}$ and $\mathcal{C}$ have the same support and if each face of $\mathcal{C}^{\prime}$ is a union of faces of $\mathcal{C}$.

Let $\mathcal{C}$ be a pure, $n$-dimensional polyhedral complex in $\mathbb{R}^{n}$. The adjacency graph $\mathcal{G}$ of $\mathcal{C}$ is the graph whose vertices are the full-dimensional faces of $\mathcal{C}$ and whose edges are the pairs of adjacent full-dimensional faces (pairs of full-dimensional faces whose intersection is a codimension-1 face). For each face $F$ of $\mathcal{C}$, the local adjacency graph of $\mathcal{C}$ at $F$ is the subgraph of $\mathcal{G}$ induced by maximal faces of $\mathcal{C}$ containing $F$. The complex $\mathcal{C}$ is locally strongly connected if, for every face $F$, the local adjacency graph of $\mathcal{C}$ at $F$ is connected. Given a complex $\mathcal{C}^{\prime}$ coarsening $\mathcal{C}$, define the edge set of $\mathcal{C}^{\prime}$ to be the set of edges $M-N$ in $\mathcal{G}$ such that $M$ and $N$ are contained in the same face of $\mathcal{C}^{\prime}$. A complex $\mathcal{C}^{\prime}$ coarsening $\mathcal{C}$ is uniquely determined

Received by the editors May 6, 2010 and, in revised form, April 14, 2011.

2010 Mathematics Subject Classification. Primary 52B99, 52C35.

The author was partially supported by NSA grant H98230-09-1-0056. 
by its edge set and vice versa. Thus to characterize complexes coarsening $\mathcal{C}$, we give a necessary and sufficient local condition for a set $\mathcal{E}$ of edges of $\mathcal{G}$ to be the edge set of a complex that coarsens $\mathcal{C}$.

Let $F$ be a codimension-2 face of $\mathcal{C}$ (often called a ridge of $\mathcal{C}$ ) and let $\mathcal{P}$ be the local adjacency graph of $\mathcal{C}$ at $F$. Let $\operatorname{Aff}(F)$ denote the affine hull of $F$, the intersection of all affine hyperplanes containing $F$. This is an affine subspace of codimension 2. Let $\operatorname{Perp}(F)$ be the unique linear subspace orthogonal to $\operatorname{Aff}(F)$. This 2 -dimensional plane is the orthogonal complement of the linear subspace $\operatorname{Aff}(F)-p$, where $p$ is any point in $\operatorname{Aff}(F)$. Choose a point $x$ in the relative interior of $F$. For each face $M$ in $\mathcal{P}$, define a cone $\{v \in \operatorname{Perp}(F): \exists \epsilon>0$ with $x+\epsilon v \in M\}$. The cones that arise in this way are the maximal cones of a fan $\left.\mathcal{C}\right|_{F}$ in the plane $\operatorname{Perp}(F)$. The adjacency graph of $\left.\mathcal{C}\right|_{F}$ is $\mathcal{P}$. A set $\mathcal{E}$ of edges in $\mathcal{G}$ has the ridge property if, for every codimension-2 face $F$ of $\mathcal{C}$, the restriction of $\mathcal{E}$ to $\mathcal{P}$ is the edge set of a fan in $\operatorname{Perp}(F)$ coarsening $\left.\mathcal{C}\right|_{F}$. We will prove the following theorem.

Theorem 1.1. Let $\mathcal{C}$ be a pure, full-dimensional, locally strongly connected polyhedral complex and let $\mathcal{G}$ be the adjacency graph on maximal faces of $\mathcal{C}$. Then a subset $\mathcal{E}$ of the edges of $\mathcal{G}$ is the edge set of a complex coarsening $\mathcal{C}$ if and only if $\mathcal{E}$ has the ridge property.

One key ingredient in the proof of Theorem 1.1 is a shortcut for proving that a collection of polyhedra is a polyhedral complex. Given a collection $\mathcal{M}$ of polyhedra, for each integer $k \geq-1$, let $\bigcap_{k}(\mathcal{M})$ be the union of all intersections $M \cap N$ such that $M, N \in \mathcal{M}$ and $\operatorname{dim}(M \cap N) \leq k$. (By convention, the empty set has dimension -1 .) For any $x \in \mathbb{R}^{n}$ and $\delta>0$, let $\mathcal{B}_{\delta}(x)$ be the open ball $\left\{y \in \mathbb{R}^{n}:|x-y|<\delta\right\}$. Although the proof of Theorem 1.1 only needs a special case of the following theorem (the case where $\mathcal{M}$ consists of $n$-dimensional polyhedra and $k=n-2$ ), it is not significantly harder to prove the more general statement.

Theorem 1.2. Fix $k \geq-1$ and let $\mathcal{M}$ be a finite collection of polyhedra in $\mathbb{R}^{n}$, each of dimension greater than $k$. Suppose:

(i) For all $x \in \operatorname{Supp}(\mathcal{M})$, there exists $\epsilon>0$ such that, for all $\delta$ with $\epsilon>\delta>0$, the set $\left[\operatorname{Supp}(\mathcal{M}) \backslash \bigcap_{k}(\mathcal{M})\right] \cap \mathcal{B}_{\delta}(x)$ is path connected.

(ii) $\quad M \cap N$ is a face of $M$ and of $N$ for all $M, N \in \mathcal{M}$ with $\operatorname{dim}(M \cap N)>k$. Then the collection of all polyhedra in $\mathcal{M}$ and their faces is a polyhedral complex.

Let $\mathcal{C}(\mathcal{A})$ be the complete polyhedral complex determined by an affine hyperplane arrangement $\mathcal{A}$. In Section 4, we prove Theorem 1.1, beginning with the special case where $\mathcal{C}$ is a pure, full-dimensional, locally strongly connected subcomplex of $\mathcal{C}(\mathcal{A})$. The proof relies on the observation, discussed in Section 3 , that every hyperplane arrangement has a property that we call path convexity. When $\mathcal{A}$ is a Coxeter arrangement, the statement that $\mathcal{A}$ is path convex is exactly the statement of Tits' solution [8, Théorème 3] to the Word Problem for the corresponding Coxeter group. Essentially equivalent observations have been made in various other settings [2, 3, 6].

Key parts of the proof of Theorem 1.1 are shared by proofs of the less general results [5, Proposition 5.2] and [4, Theorem 9]. The former, for a broad class of central hyperplane arrangements $\mathcal{A}$, uses a stronger condition than the ridge property, arising from the lattice theory of the weak order, to show that certain sets of edges of the adjacency graph $\mathcal{G}(\mathcal{A})$ are the edge sets of fans coarsening the fan $\mathcal{C}(\mathcal{A})$. 
The latter establishes Theorem 1.1 in the case $\mathcal{C}=\mathcal{C}(\mathcal{A})$, where $\mathcal{A}$ is the Coxeter arrangement for the symmetric group (i.e. the braid arrangement). For this $\mathcal{A}$, the complex $\mathcal{C}(\mathcal{A})$ is the normal fan of the permutohedron, and fans coarsening $\mathcal{C}(\mathcal{A})$ are interpreted in the language of nonparametric statistics as convex rank tests. The edge sets of fans coarsening $\mathcal{C}(\mathcal{A})$ are characterized by the square axiom and the hexagon axiom. Furthermore, edge sets satisfying the square axiom and the hexagon axiom are identified with certain conditional independence structures known as semigraphoids.

Theorem 1.1] in particular solves the problem, posed in [4, Section 1], of characterizing the edge sets of coarsenings of $\mathcal{C}(\mathcal{A})$ for arbitrary Coxeter arrangements $\mathcal{A}$. Indeed, the theorem is particularly simply stated when $\mathcal{C}=\mathcal{C}(\mathcal{A})$ for any central hyperplane arrangement $\mathcal{A}$, as we now explain.

Let $\mathbf{Z}$ be a zonotope. A set $\mathcal{E}$ of edges of $\mathbf{Z}$ has the polygon property if, for every $2 k$-gonal face $P$ of $\mathbf{Z}$, whenever $\mathcal{E}$ contains any $k-1$ consecutive edges of $P$, then $\mathcal{E}$ also contains the opposite $k-1$ consecutive edges of $P$. The polygon property on the usual permutohedron coincides with the square and hexagon axioms from 4 . When $\mathcal{A}$ is the central hyperplane arrangement dual to $\mathbf{Z}$ and $\mathcal{C}$ is $\mathcal{C}(\mathcal{A})$ (the normal fan to $\mathbf{Z}$ ), then the 2-dimensional fans $\mathcal{C}_{F}$ are defined by an arrangement of lines through the origin in $\operatorname{Perp}(F)$. Thus the ridge property reduces to the polygon property, and we have the following corollary to Theorem 1.1

Corollary 1.3. Let $\mathbf{Z}$ be a zonotope and let $\mathcal{F}$ be the normal fan of $\mathbf{Z}$. Then a set $\mathcal{E}$ of edges of $\mathbf{Z}$ is the edge set of a fan coarsening $\mathcal{F}$ if and only if $\mathcal{E}$ has the polygon property.

When $\mathcal{A}$ is a noncentral hyperplane arrangement, the analogous polygon property still characterizes coarsenings of $\mathcal{C}(A)$ for the same reason.

The proof of Theorem 1.1 also provides a local condition (Theorem 4.3) for an interior-connected union of polyhedra to be convex, which is a special case of Tietze's convexity theorem [7. (See [9, Part IV.C].) In Section 5] we extend all of our results to the context of oriented matroids, proving, in particular, an oriented matroid version (Theorem 5.5) of Tietze's convexity theorem.

\section{Polyhedral complexes}

In this section, we prove Theorem 1.2, We begin by establishing a well-known easier result. (See, for example, [4, Lemma 14] or [5, Lemma 3.2].)

Lemma 2.1. Let $\mathcal{M}$ be a finite collection of polyhedra and let $\mathcal{C}$ be the collection consisting of all polyhedra in $\mathcal{M}$ and their faces. Suppose $M \cap N$ is a face of $M$ and of $N$ for all $M, N \in \mathcal{M}$. Then $\mathcal{C}$ is a polyhedral complex.

Proof. Let $F, G, M$, and $N$ be polyhedra in $\mathcal{C}$ such that $F$ is a face of $M$ and $G$ is a face of $N$. We claim that $F \cap G$ is a face of $M \cap N$. If $F=M$ and $G=N$, then the assertion is trivial, so without loss of generality, $F$ is a proper face of $M$. Let $H$ be a hyperplane such that $H \cap M$ is the face $F$ of $M$. Then also $H \cap(M \cap N)=F \cap N$ is a face of $M \cap N$. If $G=N$, then $F \cap G$ is a face of $M \cap N$, and if not, we argue similarly that $M \cap G$ is a face of $M \cap N$. Thus $F \cap G=(F \cap N) \cap(M \cap G)$ is a face of $M \cap N$, and we have proven the claim in either case. Now, since $F$ and $F \cap G$ are faces of $M \cap N, F \cap G$ is a face of $F$. Symmetrically, $F \cap G$ is a face of $G$. 
Proof of Theorem 1.2. We will verify the hypotheses of Lemma 2.1. Let $M$ and $N$ be distinct polyhedra in $\mathcal{M}$, let $F$ be the polyhedron $M \cap N$, and let $d$ be the dimension of $F$. If $F=\emptyset$, then we are done. Otherwise, there exists a point $x \in F$ such that $x$ is not contained in any polyhedron in $\mathcal{C}$ of dimension strictly less than $d$. By hypothesis (i), there exists $\epsilon>0$ such that, for all $\delta$ with $\epsilon>\delta>0$, the set $\left(\operatorname{Supp}(\mathcal{M}) \backslash \bigcap_{k}(\mathcal{M})\right) \cap \mathcal{B}_{\delta}(x)$ is path connected. Every face of $\mathcal{C}$ not containing $x$ is some positive distance from $x$ and there are finitely many faces of $\mathcal{C}$. Thus there exists $\delta$ with $\epsilon>\delta>0$ such that every face of $\mathcal{C}$ intersecting $\mathcal{B}_{\delta}(x)$ actually contains $x$.

Now $x$ is in $M$ and in $N$, so $\mathcal{B}_{\delta}(x)$ intersects the relative interiors of $M$ and $N$. Let $y \in\left(\mathcal{B}_{\delta}(x) \cap \operatorname{relint}(M)\right) \backslash \bigcap_{k}(\mathcal{M})$ and $z \in\left(\mathcal{B}_{\delta}(x) \cap \operatorname{relint}(N)\right) \backslash \bigcap_{k}(\mathcal{M})$. Let $\alpha:[0,1] \rightarrow\left(\operatorname{Supp}(\mathcal{M}) \backslash \bigcap_{k}(\mathcal{M})\right) \cap \mathcal{B}_{\delta}(x)$ be a path from $y$ to $z$. We will use $\alpha$ to construct a sequence $M=M_{0}, \ldots, M_{j}=N$ of polyhedra in $\mathcal{M}$ such that, for each $i=1, \ldots, j$, the intersection $M_{i-1} \cap M_{i}$ is of dimension greater than $k$. The set $M$ is closed, so $\alpha^{-1}(M)$ is a closed subset of $[0,1]$. If $t_{1}$ is the maximum of the set $\alpha^{-1}(M)$, then $\alpha\left(t_{1}\right)$ is in $M$ and in some $M_{1} \in \mathcal{M}$. But $\alpha\left(t_{1}\right) \in \operatorname{Supp}(\mathcal{M}) \backslash \bigcap_{k}(\mathcal{M})$, so $M \cap M_{1}$ has dimension greater than $k$. If $M_{1} \neq N$, then repeat the construction to find $M_{2} \in \mathcal{M}$ such that $\operatorname{dim}\left(M_{1} \cap M_{2}\right)>k$, and continue until $M_{j}=N$.

We now show that for any sequence $M_{0}, \ldots, M_{j}$ of polyhedra in $\mathcal{M}$ such that $M_{i-1} \cap M_{i}$ is of dimension greater than $k$ for each $i=1, \ldots, j$, the intersection $M_{0} \cap \cdots \cap M_{j}$ is a face of $M_{j}$. We argue by induction on $j$, the case $j=0$ being trivial. If $j>0$, then by induction $M_{0} \cap \cdots \cap M_{j-1}$ is a face $G$ of $M_{j-1}$. Now $G^{\prime}=M_{j-1} \cap M_{j}$ is a face of $M_{j-1}$ and of $M_{j}$ by hypothesis (ii). Thus $M_{0} \cap \cdots \cap M_{j}$ is a face of $M_{j-1}$ because it is the intersection of two faces, $G$ and $G^{\prime}$, of $M_{j-1}$. But then $M_{0} \cap \cdots \cap M_{j}$ is a face of $G^{\prime}$, and thus a face of $M_{j}$.

We have shown that $F^{\prime}=M_{0} \cap \cdots \cap M_{j}$ is a face of $N$. Since $\alpha$ is contained in $\mathcal{B}_{\delta}(x)$, each $M_{i}$ intersects $\mathcal{B}_{\delta}(x)$, so by the definition of $\delta$, each $M_{i}$ contains $x$. Thus $F^{\prime}$ contains $x$, so by the definition of $x, F^{\prime}$ is a face of $\mathcal{C}$ of dimension at least $d$. However, $F^{\prime}$ is contained in the $d$-dimensional polyhedron $F=M \cap N$, so $F^{\prime}$ has dimension $d$. Let $H$ be a hyperplane such that $N \cap H$ is $F^{\prime}$. Since $F^{\prime} \subseteq F$ and both are $d$-dimensional polyhedra, we have $F \subseteq H$. Thus $F \subseteq(N \cap H)=F^{\prime}$. Therefore $F^{\prime}=F$, so that $F$ is a face of $N$. By symmetry, $F$ is a face of $M$.

\section{Path CONVEXity}

In this section, we show that every hyperplane arrangement has a property that we call path convexity. This fact will be crucial in the proof of Theorem 1.1.

A hyperplane arrangement in $\mathbb{R}^{n}$ is a finite collection $\mathcal{A}$ of affine hyperplanes. The closures of the connected components of $\mathbb{R}^{n} \backslash\left(\bigcup_{H \in \mathcal{A}} H\right)$ are called regions. The regions are the maximal faces of a complete polyhedral complex $\mathcal{C}(\mathcal{A})$. Let $\mathcal{G}(\mathcal{A})$ be the adjacency graph of the complex $\mathcal{C}(\mathcal{A})$.

Let $Q, R \in \mathcal{R}(\mathcal{A})$. A path in $\mathcal{G}(\mathcal{A})$ from $Q$ to $R$ is a sequence $R_{0}, R_{1}, \ldots, R_{k}$ of regions with $Q=R_{0}$ and $R=R_{k}$, such that $R_{i-1}-R_{i}$ is an edge in $\mathcal{G}(\mathcal{A})$ for each $i$ from 1 to $k$. The length of a path $R_{0}, R_{1}, \ldots, R_{k}$ is $k$, one less than the number of entries in the sequence. A braid move on a path alters the path by deleting an adjacent subsequence $Q_{0}, \ldots, Q_{m}$ from the path and replacing it with a sequence $Q_{0}^{\prime}, \ldots, Q_{m}^{\prime}$ such that $Q_{0}=Q_{0}^{\prime}, Q_{m}=Q_{m}^{\prime}$ and the cycle $Q_{0}, Q_{1}, \ldots, Q_{m} Q_{m-1}^{\prime}, \ldots, Q_{0}^{\prime}$ is a polygon in $\mathcal{G}(\mathcal{A})$. A braid move does not change 
the length of the path. A nil move on paths alters a path by replacing an adjacent subsequence $Q_{0}, Q_{1}, Q_{2}$ such that $Q_{0}=Q_{2}$ by the singleton sequence $Q_{0}$.

We say that a path $\gamma$ is reduced if it has minimal length among all paths from $Q$ to $R$. The arrangement $\mathcal{A}$ is path convex if, for every pair $Q, R$ of regions in $\mathcal{A}$, every path $\gamma$ from $Q$ to $R$, and every reduced path $\rho$ from $Q$ to $R$, the path $\gamma$ can be transformed, by a sequence of braid moves and nil moves, to the path $\rho$. The appropriateness of the term "convex" in this definition will become apparent in the proof of Theorem 1.1, particularly in Lemma 4.1.

Theorem 3.1. Every hyperplane arrangement is path convex.

A slightly weaker statement for oriented matroids is [1, Proposition 4.4.6]. We now prepare to prove Theorem 3.1. Given $Q, R \in \mathcal{R}(\mathcal{A})$, let $S(Q, R)$ be the set of hyperplanes of $\mathcal{A}$ that separate $Q$ from $R$. The following lemma is well known.

Lemma 3.2. A path from $Q$ to $R$ is reduced if and only if its length is $|S(Q, R)|$.

Proof. Moving from one region to an adjacent region, one crosses exactly one hyperplane of $\mathcal{A}$. Thus a path from $Q$ to $R$ has length at least $|S(Q, R)|$. If $x$ is a generic point in the interior of $Q$ and $y$ is a generic point in the interior of $R$, then the line segment $\overline{x y}$ intersects each hyperplane in $S(Q, R)$ exactly once, intersects no two hyperplanes in $S(Q, R)$ in the same point, and intersects no hyperplane of $\mathcal{A} \backslash S(Q, R)$. Thus $\overline{x y}$ defines a path of length $|S(Q, R)|$ from $Q$ to $R$.

The arrangement $\mathcal{A}$ is reduced-path connected if, for every pair $Q, R$ of regions in $\mathcal{A}$ and every pair $\gamma, \rho$ of reduced paths from $Q$ to $R$, the path $\gamma$ can be transformed, by a sequence of braid moves, to the path $\rho$.

Lemma 3.3. If $\mathcal{A}$ is reduced-path connected, then $\mathcal{A}$ is path convex.

Proof. Suppose $\mathcal{A}$ is reduced-path connected. Let $\gamma=\left(R_{0}, R_{1}, \ldots, R_{m}\right)$ be any path from $Q$ to $R$ and let $\rho$ be any reduced path from $Q$ to $R$.

If $\gamma$ is not reduced, then Lemma 3.2 says that $m>|S(P, Q)|$. Thus there exists a smallest positive integer $k$ such that $k>\left|S\left(R_{0}, R_{k}\right)\right|$. Then $R_{0}, R_{1}, \ldots, R_{k-1}$ is a reduced path and $\left|S\left(R_{0}, R_{k}\right)\right|=k-2$. By Lemma 3.2. there is a reduced path $R_{0}^{\prime}, R_{1}^{\prime}, \ldots, R_{k-2}^{\prime}$ from $R_{0}$ to $R_{k}$, and thus the path $R_{0}^{\prime}, R_{1}^{\prime}, \ldots, R_{k-2}^{\prime}, R_{k-1}$ is reduced. (Notice that an unprimed $R_{k-1}$ is the last region in this path.) Since $\mathcal{A}$ is reduced-path connected, there is a sequence of braid moves that transforms $R_{0}, R_{1}, \ldots, R_{k-1}$ to $R_{0}^{\prime}, R_{1}^{\prime}, \ldots, R_{k-2}^{\prime}, R_{k-1}$. The same braid moves transform the path $\gamma$ to $R_{0}^{\prime}, R_{1}^{\prime}, \ldots, R_{k-2}^{\prime}, R_{k-1}, R_{k}, \ldots, R_{m}$. But $R_{k-2}^{\prime}=R_{k}$, so a nil move can be applied to $R_{0}^{\prime}, R_{1}^{\prime}, \ldots, R_{k-2}^{\prime}, R_{k-1}, R_{k}, \ldots, R_{m}$, replacing $R_{k-2}^{\prime}, R_{k-1}, R_{k}$ with $R_{k}$.

Repeating the process, we transform $\gamma$ to a reduced path $\gamma^{\prime}$ by a sequence of braid moves and nil moves. By the reduced-path connectedness of $\mathcal{A}, \gamma^{\prime}$ can be transformed to $\rho$ by a sequence of braid moves.

Lemma 3.3 reduces Theorem 3.1 to the following theorem.

Theorem 3.4. Every hyperplane arrangement is reduced-path connected.

Theorem 3.4 was proved by Deligne [3, Proposition 1.12] for simplicial hyperplane arrangements, by Salvetti [6, Lemma 11], and also by Cordovil and Moreira [2, Theorem 2.4] for oriented matroids. For the sake of completeness, we give a short proof which is similar to the argument given in [2, 6]. 
Proof of Theorem 3.4. Let $\gamma=\left(Q_{0}, Q_{1}, \ldots, Q_{k}\right)$ and $\rho=\left(R_{0}, R_{1}, \ldots, R_{k}\right)$ be reduced paths with $Q_{0}=R_{0}$ and $Q_{k}=R_{k}$. We will show that $\gamma$ and $\rho$ are related by a sequence of braid moves.

Let $F_{0}, F_{1}, \ldots, F_{m}$ be a sequence of facets (maximal proper faces) of $Q_{0}$, chosen to minimize $m$ subject to the following requirements: (1) that $F_{0}=Q_{0} \cap Q_{1}$, (2) that $F_{m}=Q_{0} \cap R_{1}$, (3) that $F_{i-1} \cap F_{i}$ has codimension 2 for each $i=1, \ldots, m$, and (4) that, for each $i=0,1, \ldots, m$, the hyperplane $H_{i}$ containing $F_{i}$ is in the set $S\left(Q_{0}, Q_{k}\right)$. We will show that such a sequence $F_{0}, F_{1}, \ldots, F_{m}$ exists. For each $i=1, \ldots, k$, choose a point $x_{i} \in Q_{i-1} \cap Q_{i}$ and a point $y_{i} \in R_{i-1} \cap R_{i}$ and concatenate the segments $\overline{x_{1} x_{2}}, \ldots, \overline{x_{k-1} x_{k}}, \overline{x_{k} y_{k}}$, and $\overline{y_{k} y_{k-1}}, \ldots, \overline{y_{2} y_{1}}$ to construct a continuous curve $\alpha:[0,1] \rightarrow \mathbb{R}^{n}$ that begins in $Q_{0} \cap Q_{1}$, passes through $Q_{1}, \ldots, Q_{k}, R_{k-1}, \ldots, R_{1}$, ending in $Q_{0} \cap R_{1}$. Choose a point $x$ in the relative interior of $Q_{0}$. Define a continuous curve $\beta$ in the boundary of $Q_{0}$ by taking $\beta(t)$ to be the unique point on the boundary of $Q_{0}$ and on the line segment with endpoints $x$ and $\alpha(t)$. Let $U$ be the union of all lines that contain $x$ and that intersect a face of $Q_{0}$ of codimension 3 or greater. Since $U$ has codimension 2, for generic choices of the $x_{i}$ and $y_{i}$, the path $\alpha$ avoids $U$. Thus $\beta$ avoids faces of $Q_{0}$ of codimension 3 or greater, so $\beta$ defines a sequence $F_{0}, F_{1}, \ldots, F_{m}$ of facets of $Q_{0}$ satisfying requirements (1), (2), and (3). To see that the sequence satisfies requirement (4), note that each $H_{i}$ intersects a line segment connecting a point in the interior of some $Q_{j}$ or $R_{j}$, for $1 \leq j \leq k$, to the point $x \in \operatorname{int}\left(Q_{0}\right)$. Thus $H_{i} \in S\left(Q_{0}, Q_{j}\right)$ or $H \in S\left(Q_{0}, R_{j}\right)$ for some $1 \leq j \leq k$. But since $\gamma$ and $\rho$ are reduced paths, we have $S\left(Q_{0}, Q_{1}\right) \subset S\left(Q_{0}, Q_{2}\right) \subset \cdots \subset S\left(Q_{0}, Q_{k}\right)$, and $S\left(Q_{0}, R_{1}\right) \subset S\left(Q_{0}, R_{2}\right) \subset \cdots \subset S\left(Q_{0}, R_{k}\right)$, so $H_{i} \in S\left(Q_{0}, Q_{k}\right)$.

We now argue by induction on $k$ and on $m$. If $k=0$, then $Q_{0}=Q_{k}$ and the assertion is trivial. Now suppose $k>0$. If $m=0$, then $F_{0}=F_{m}$, so $Q_{1}=R_{1}$. By induction on $k$, there is a sequence of braid moves relating $Q_{1}, \ldots, Q_{k}$ to $R_{1}, \ldots, R_{k}$. This same sequence of braid moves relates $\gamma$ to $\rho$.

Now suppose $m>0$ as well. Let $\mathcal{A}^{\prime}$ be the set of hyperplanes in $\mathcal{A}$ containing the codimension-2 face $F_{m-1} \cap F_{m}$. Any region $T$ with $\left\{H_{m-1}, H_{m}\right\} \subseteq S\left(Q_{0}, T\right)$ has $\mathcal{A}^{\prime} \subseteq S\left(Q_{0}, T\right)$. In particular, $\mathcal{A}^{\prime} \subseteq S\left(Q_{0}, Q_{k}\right)$. Let $x$ be a point in the relative interior of $Q_{0}$ and let $p$ be a point in the relative interior of $F_{m-1} \cap F_{m}$. For small enough $\epsilon>0$, the point $p+\epsilon(p-x)$ is in a region $T$ with $S\left(Q_{0}, T\right)=\mathcal{A}^{\prime}$. Let $\mu$ be a reduced path from $T$ to $Q_{k}$. Then $\mu$ has length $\left|S\left(Q_{0}, Q_{k}\right)\right|-\left|\mathcal{A}^{\prime}\right|$ because $S\left(T, Q_{k}\right)=S\left(Q_{0}, Q_{k}\right) \backslash \mathcal{A}^{\prime}$. There are two reduced paths from $Q_{0}$ to $T$, related by a braid move involving the polygon dual to $F_{m-1} \cap F_{m}$. Concatenating these paths with $\mu$, we obtain a reduced path $\rho^{\prime}$ from $Q_{0}$ to $Q_{k}$ starting with the regions $Q_{0}, R_{1}$, and a reduced path $\gamma^{\prime}$ from $Q_{0}$ to $Q_{k}$ starting with $Q_{0}$ and then continuing to the region which shares the facet $F_{m-1}$ with $Q_{0}$. By induction on $m$, the paths $\gamma$ and $\gamma^{\prime}$ are related by a sequence of braid moves. By construction, $\gamma^{\prime}$ and $\rho^{\prime}$ are related by a single braid move. Let $\rho^{\prime}=R_{0}^{\prime}, R_{1}^{\prime}, \ldots, R_{k}^{\prime}$, so that $R_{0}^{\prime}=R_{0}=Q_{0}$, $R_{1}^{\prime}=R_{1}$, and $R_{k}^{\prime}=R_{k}=Q_{k}$. By induction on $k, R_{1}^{\prime}, \ldots, R_{k}^{\prime}$ and $R_{1}, \ldots, R_{k}$ are related by a sequence of braid moves, so $\rho^{\prime}$ and $\rho$ are related by the same sequence of braid moves. We have found a sequence of braid moves relating $\gamma$ and $\rho$.

\section{EdGe SETS OF COARSENINGS}

In this section, we prove Theorem 1.1. One direction of the theorem is easy. Indeed, suppose that $\mathcal{C}^{\prime}$ is a complex coarsening $\mathcal{C}$. If the edge set of $\mathcal{C}^{\prime}$ fails the 
ridge property at some codimension- 2 face $F$ of $\mathcal{C}$, then we reach a contradiction to the supposition that $\mathcal{C}^{\prime}$ is a complex: Either some maximal face of $\mathcal{C}^{\prime}$ is not convex or there is a pair of maximal faces $C$ and $D$ of $\mathcal{C}^{\prime}$, each having $F$ in their boundary, such that $C \cap D$ is not a face of $C$. This contradiction proves the "only if" assertion of Theorem 1.1

Let $\mathcal{A}$ be a hyperplane arrangement and continue the notation of Section 3 , We first prove Theorem 1.1 in the special case where $\mathcal{C}$ is a pure, full-dimensional, locally strongly connected subcomplex of $\mathcal{C}(\mathcal{A})$. Recall that in Section 1 we defined the polygon property for a set $\mathcal{E}$ of edges of $\mathcal{G}(\mathcal{A})$ when $\mathcal{A}$ is a central arrangement and pointed out that the polygon property is equivalent to the ridge property in this case. We now generalize the polygon property by allowing $\mathcal{A}$ to be noncentral and by allowing $\mathcal{C}$ to be a subcomplex of $\mathcal{C}(\mathcal{A})$.

Let $F$ be a codimension-2 face of $\mathcal{C}(\mathcal{A})$. The polygon in $\mathcal{G}(\mathcal{A})$ associated to $F$ is the cycle $\mathcal{P}$ in $\mathcal{G}(\mathcal{A})$ consisting of all of the full-dimensional faces of $\mathcal{C}(\mathcal{A})$ containing $F$. Every polygon in $\mathcal{G}(\mathcal{A})$ is a $2 k$-gon for some $k \geq 2$. Let $\mathcal{C}$ be a pure, full-dimensional, locally strongly connected subcomplex of $\mathcal{C}(\mathcal{A})$ with adjacency graph $\mathcal{G}$. A set $\mathcal{E}$ of edges of $\mathcal{G}$ has the polygon property if the following condition holds for every $2 k$-gon $\mathcal{P}$ in $\mathcal{G}(\mathcal{A})$ : If $\mathcal{E}$ contains any $k-1$ consecutive edges of $\mathcal{P}$, then either the opposite $k$ vertices of $\mathcal{P}$ are not vertices of $\mathcal{G}$ or $\mathcal{E}$ also contains the opposite $k-1$ consecutive edges of $\mathcal{P}$. (It is important to keep in mind that the polygon property for $\mathcal{E}$ is defined in terms of polygons in $\mathcal{G}(\mathcal{A})$, not in $\mathcal{G}$.) It is immediate that the ridge property is equivalent to the polygon property in the case where $\mathcal{C}$ is a pure, full-dimensional subcomplex of $\mathcal{C}(\mathcal{A})$.

A set $\mathcal{E}$ of edges of $\mathcal{G}$ has the weak polygon property if the following condition holds for each $2 k$-gon $\mathcal{P}$ in $\mathcal{G}(\mathcal{A})$ : If $\mathcal{E}$ contains any $k$ consecutive edges of $\mathcal{P}$, then $\mathcal{E}$ contains all of the edges of $\mathcal{P}$. The polygon property implies the weak polygon property.

A pre-complex $\mathcal{C}^{\prime}$ is a collection of polyhedra such that if $F \in \mathcal{C}^{\prime}$ and $G$ is a face of $F$, then $G \in \mathcal{C}^{\prime}$. A pre-complex $\mathcal{C}^{\prime}$ coarsens a complex $\mathcal{C}$ if $\mathcal{C}^{\prime}$ and $\mathcal{C}$ have the same support and if each face of $\mathcal{C}^{\prime}$ is a union of faces of $\mathcal{C}$. (This matches the definition given earlier when $\mathcal{C}^{\prime}$ was assumed to be a complex.) We first show that the weak polygon property characterizes pre-complexes coarsening a full-dimensional subcomplex $\mathcal{C}$ of $\mathcal{C}(\mathcal{A})$.

Lemma 4.1. Let $\mathcal{C}$ be a full-dimensional subcomplex of $\mathcal{C}(\mathcal{A})$ and let $\mathcal{E}$ be a set of edges in $\mathcal{G}$ with the weak polygon property. Then $\mathcal{E}$ is the edge set of a pre-complex $\mathcal{C}^{\prime}$ coarsening $\mathcal{C}$. The maximal faces of $\mathcal{C}^{\prime}$ are full-dimensional and have pairwise disjoint interiors.

Proof. We will think of $\mathcal{E}$ not only as a set of edges of $\mathcal{G}$, but as a graph in its own right, whose vertices are the full-dimensional faces of $\mathcal{C}$. Each connected component of $\mathcal{E}$ is in particular a set of regions. Consider the union of this set of regions. We must show that each such union is a polyhedron. In fact, we need only show convexity; the fact that the union is a polyhedron will follow.

Let $M$ be a such a union. Choose points $x, y \in M$. We will show that the line segment $\overline{x y}$ is contained in $M$. If there is a region $R$ of $\mathcal{A}$ with $\{x, y\} \subseteq R \subseteq M$, then $\overline{x y} \subseteq R \subseteq M$, so suppose $x$ and $y$ are not contained in the same region. Let $Q$ be a region with $x \in Q \subseteq M$ and let $R$ be a region with $y \in R \subseteq M$. Suppose for 
the moment that $x \in \operatorname{int}(Q)$ and $y \in \operatorname{int}(R)$ and that the line segment $\overline{x y}$ does not intersect any face of $\mathcal{C}(\mathcal{A})$ of codimension greater than 1. Then, as in the proof of Lemma 3.2 $\overline{x y}$ defines a path $\rho$ of length $|S(Q, R)|$ from $Q$ to $R$. By Lemma 3.2, $\rho$ is a reduced path. On the other hand, since $Q$ and $R$ are both contained in $M$, there is a path $\gamma=\left(R_{0}, R_{1}, \ldots, R_{k}\right)$ from $Q$ to $R$ which is not only a path in $\mathcal{G}(\mathcal{A})$, but also a path in $\mathcal{E}$. By the path convexity of $\mathcal{A}$, the path $\gamma$ can be converted to the path $\rho$ by a sequence of braid moves and nil moves. Trivially, each nil move applied to $\gamma$ produces a new path in $\mathcal{E}$. Furthermore, the weak polygon property of $\mathcal{E}$ implies that, when a braid move is performed on $\gamma$, the new path is also a path in $\mathcal{E}$. We conclude that $\rho$ is a path in $\mathcal{E}$. In particular, each region in $\rho$ is contained in $M$, so $\overline{x y} \subseteq M$.

If $x \notin \operatorname{int}(Q)$, if $y \notin \operatorname{int}(R)$ and/or if $\overline{x y}$ intersects a face of $\mathcal{C}(\mathcal{A})$ of codimension greater than 1 , then there exist points $x^{\prime} \in \operatorname{int}(Q)$ and $y^{\prime} \in \operatorname{int}(R)$, arbitrarily close to $x$ and $y$ respectively, such that $\overline{x^{\prime} y^{\prime}}$ does not intersect any face of $\mathcal{C}(\mathcal{A})$ of codimension greater than 1 . Therefore each point on $\overline{x y}$ is arbitrarily close to a point which we have proven to be in $M$. Since $M$ is a union of finitely many closed polyhedra, it is closed, so $\overline{x y} \subseteq M$.

The collection of all such polyhedra $M$ and their nonempty faces is a precomplex $\mathcal{C}^{\prime}$ coarsening $\mathcal{C}(\mathcal{A})$. By construction, the maximal faces of $\mathcal{C}^{\prime}$ are fulldimensional and have pairwise disjoint interiors. It remains to show that $\mathcal{E}$ is indeed the edge set of $\mathcal{C}^{\prime}$. By construction, $\mathcal{E}$ is contained in the edge set of $\mathcal{C}^{\prime}$. Suppose $\{Q, R\}$ is a pair of regions contained in the same maximal face of $\mathcal{C}^{\prime}$. Then the path $Q, R$ is a reduced path $\rho$ (in $\mathcal{G}(\mathcal{A})$ ) from $Q$ to $R$. Since $Q$ and $R$ are contained in the same maximal face of $\mathcal{C}^{\prime}$, there exists a path $\gamma$ in $\mathcal{E}$ from $Q$ to $R$. Arguing as above, the path $\rho$ is also a path in $\mathcal{E}$, or in other words, $Q-R$ is an edge in $\mathcal{E}$. We have shown that $\mathcal{E}$ is the edge set of $\mathcal{C}^{\prime}$.

Lemma 4.2. Let $\mathcal{C}$ be a full-dimensional subcomplex of $\mathcal{C}(\mathcal{A})$. Let $\mathcal{E}$ be a set of edges in $\mathcal{G}$ with the polygon property, so that $\mathcal{E}$ is the edge set of a pre-complex $\mathcal{C}^{\prime}$ coarsening $\mathcal{C}$, by Lemma 4.1. If $M$ and $N$ are maximal faces of $\mathcal{C}^{\prime}$ and $M \cap N$ has codimension 1, then $M \cap N$ is a face of $M$ and a face of $N$.

Proof. Suppose $M$ and $N$ are maximal faces of $\mathcal{C}^{\prime}$ and $M \cap N$ has codimension 1. Since $M$ and $N$ are unions of faces of $\mathcal{C}(\mathcal{A})$, their intersection is a union of codimension- 1 faces of $\mathcal{C}(\mathcal{A})$. Since $M$ and $N$ are convex and have disjoint interiors, $M \cap N$ is contained in some face $F$ of $M$ of codimension 1. The face $F$ is also a union of codimension-1 faces of $\mathcal{C}(\mathcal{A})$. We now prove the following claim: If $G$ is a codimension-1 face of $\mathcal{C}(\mathcal{A})$ contained in $M \cap N$ and $G^{\prime}$ is a codimension-1 face of $\mathcal{C}(\mathcal{A})$ contained in $F$ such that $G \cap G^{\prime}$ has codimension 2 , then $G^{\prime} \subseteq(M \cap N)$.

To prove the claim, let $\mathcal{P}$ be the polygon consisting of regions containing the codimension-2 face $G \cap G^{\prime}$ of $\mathcal{C}(\mathcal{A})$. Since $G$ and $G^{\prime}$ are both in the face $F$ of $M$, the polygon $\mathcal{P}$ contains a region $R$ with $G \subset R \subset M$ and a region $R^{\prime}$ with $G^{\prime} \subset R^{\prime} \subset M$. Since $G$ and $G^{\prime}$ are both contained in the hyperplane defining $F$ as a codimension-1 face of $M$, we can name the regions of $\mathcal{P}$ as the cycle $R_{0}, R_{1}, \ldots, R_{2 k}=R_{0}$ with $R_{1}=R$ and $R_{k}=R^{\prime}$. Since $R_{1}$ and $R_{k}$ are both in $M$, and since $M$ is convex, we conclude that the regions $R_{1}, \ldots, R_{k}$ are all in $M$. Now the polygon property implies that the regions $R_{k+1}, \ldots, R_{2 k}$ are all in the same maximal face of $\mathcal{C}^{\prime}$. But $R_{2 k} \subset N$ because $G \subset N$. Thus in particular $R_{k+1} \subset N$, so that $G^{\prime} \subset N$. By hypothesis, $G^{\prime} \subset F \subset M$, so $G^{\prime} \subset M \cap N$. We have established the claim. 
Given any two codimension- 1 faces $G$ and $G^{\prime}$ of $\mathcal{C}(\mathcal{A})$ contained in $F$, choosing generic points $x \in G$ and $x^{\prime} \in G^{\prime}$, the line segment $\overline{x x^{\prime}}$ defines a sequence $G=$ $G_{0}, G_{1}, \ldots, G_{k}=G^{\prime}$ of codimension-1 faces of $\mathcal{C}(\mathcal{A})$, contained in $F$, such that $G_{i-1}$ and $G_{i}$ share a codimension-2 face for each $i=1, \ldots, k$. Since there exists a codimension-1 face of $\mathcal{C}(\mathcal{A})$ in $M \cap N$, the claim and a simple induction on $k$ establish that $F \subseteq M \cap N$. Thus $F=M \cap N$, so that $M \cap N$ is a face of $M$. By symmetry, $M \cap N$ is a face of $N$ as well.

Suppose $\mathcal{C}$ is a pure, full-dimensional, locally strongly connected subcomplex of $\mathcal{C}(\mathcal{A})$, and suppose $\mathcal{E}$ is a set of edges of $\mathcal{G}$ having the polygon property. Lemma 4.1 says that $\mathcal{E}$ is the edge set of a pure, full-dimensional pre-complex $\mathcal{C}^{\prime}$ coarsening $\mathcal{C}$ and that the set $\mathcal{M}$ of maximal faces of $\mathcal{C}^{\prime}$ consists of $n$-dimensional polytopes with pairwise disjoint interiors. Lemma 4.2 says that $M \cap N$ is a face of $M$ and of $N$ for all pairs $M, N \in \mathcal{M}$ such that $\operatorname{dim}(M \cap N)>n-2$. We now show that $\mathcal{M}$ satisfies condition (i) of Theorem 1.2 with $k=n-2$. Let $x$ be a point in $\operatorname{Supp}(\mathcal{M})=\operatorname{Supp}(\mathcal{C})$ and let $F$ be the face of $\mathcal{C}$ such that $x \in \operatorname{relint}(F)$. Since $\mathcal{C}$ is locally strongly connected, by considering the local adjacency graph of $\mathcal{C}$ at $F$, we see that there exists $\epsilon>0$ such that $\operatorname{Supp}(\mathcal{M}) \cap \mathcal{B}_{\delta}(x)$ has a connected interior for all $\delta$ with $\epsilon>\delta>0$. Then since $\bigcap_{n-2}(\mathcal{M})$ is at most $(n-2)$-dimensional, the set $\left[\operatorname{Supp}(\mathcal{M}) \backslash \bigcap_{n-2}(\mathcal{M})\right] \cap \mathcal{B}_{\delta}(x)$ is path connected. Theorem 1.2 completes the proof of Theorem 1.1 for this special choice of $\mathcal{C}$.

Now let $\mathcal{C}$ be any pure, full-dimensional, locally strongly connected polyhedral complex and let $\mathcal{A}$ be the arrangement of hyperplanes consisting of hyperplanes containing codimension-1 faces of $\mathcal{C}$. Then every polyhedron in $\mathcal{C}$ is a union of faces of $\mathcal{C}(\mathcal{A})$. Let $\overline{\mathcal{C}}$ be the subcomplex of $\mathcal{C}(\mathcal{A})$ consisting of faces contained in $\operatorname{Supp}(\mathcal{C})$. Then $\mathcal{C}$ coarsens $\overline{\mathcal{C}}$. It is easily verified that $\overline{\mathcal{C}}$ is locally strongly connected. (Local adjacency graphs of $\mathcal{C}$ are obtained from local adjacency graphs of $\overline{\mathcal{C}}$ by contracting edges.)

Continue the notation $\mathcal{G}$ for the adjacency graph on maximal faces of $\mathcal{C}$, and let $\overline{\mathcal{G}}$ be the adjacency graph on full-dimensional faces of $\overline{\mathcal{C}}$. Let $\mathcal{E}$ be a set of edges of $\mathcal{G}$ with the ridge property. We now define a set $\overline{\mathcal{E}}$ of edges of $\overline{\mathcal{G}}$, describing $\overline{\mathcal{E}}$ as a set of codimension- 1 faces of $\overline{\mathcal{C}}$. Each such face represents the pair of regions containing it. Let $\bar{F}$ be a codimension-1 face of $\overline{\mathcal{C}}$, not contained in the boundary of $\operatorname{Supp}(\mathcal{C})$. If $\bar{F}$ is contained in a codimension- 1 face $F$ of $\mathcal{C}$, then choose $\bar{F}$ to be an edge in $\overline{\mathcal{E}}$ if and only if $F$ is an edge in $\mathcal{E}$. Otherwise, since $\mathcal{C}$ coarsens $\overline{\mathcal{C}}, \bar{F}$ is contained in a full-dimensional face of $\mathcal{C}$ and intersects the interior of that full-dimensional face. In this case, choose $\bar{F}$ to be an edge in $\overline{\mathcal{E}}$.

Let $\bar{F}$ be a codimension-2 face of $\overline{\mathcal{C}}$. If $\bar{F}$ is contained in a codimension-2 face $F$ of $\mathcal{C}$, then $\operatorname{Aff}(\bar{F})$ and $\operatorname{Aff}(F)$ coincide. Since $\mathcal{E}$ has the ridge property, it restricts to the edge set of a fan in $\operatorname{Perp}(F)$ which coarsens $\left.\mathcal{C}\right|_{F}$. But $\overline{\mathcal{E}}$ defines the same fan in $\operatorname{Perp}(\bar{F})=\operatorname{Perp}(F)$, so $\overline{\mathcal{E}}$ has the ridge property at $\bar{F}$. If $\bar{F}$ is contained in a codimension- 1 face $F$ of $\mathcal{C}$ and intersects the relative interior of $F$, then there are two cases, depending on whether $F$ is in the interior of $\operatorname{Supp}(\mathcal{C})$ or on the boundary of $\operatorname{Supp}(\mathcal{C})$. In either case, it is immediate that the restriction of $\overline{\mathcal{E}}$ is the edge set of a fan coarsening $\left.\overline{\mathcal{C}}\right|_{\bar{F}}$. In one case, the fan has two maximal cones, which are half-planes, and in the other case, the fan has a unique maximal cone, which is a half-plane. Since $\mathcal{C}$ coarsens $\overline{\mathcal{C}}$, the only possibility remaining is that $\bar{F}$ is contained in, and intersects the interior of, a full-dimensional face $F$ of $\mathcal{C}$. In this case, the 
restriction of $\overline{\mathcal{E}}$ defines the fan having the whole plane as its unique nonempty face. In all cases, $\overline{\mathcal{E}}$ has the ridge property at $\bar{F}$. Since $\bar{F}$ was chosen arbitrarily, $\overline{\mathcal{E}}$ has the ridge property.

By the special case of Theorem 1.1 already proved, $\overline{\mathcal{E}}$ is the edge set of a complex $\mathcal{C}^{\prime}$ coarsening $\overline{\mathcal{C}}$. For each maximal face $M$ of $\mathcal{C}$, every pair of adjacent regions of $\mathcal{A}$ contained in $M$ is an edge in $\overline{\mathcal{E}}$, so $\mathcal{C}^{\prime}$ coarsens $\mathcal{C}$ as well. We will complete the proof by showing that $\mathcal{E}$ is the edge set of $\mathcal{C}^{\prime}$, as a coarsening of $\mathcal{C}$. Let $M$ and $N$ be adjacent maximal faces of $\mathcal{C}$. If $M-N$ is an edge in $\mathcal{E}$, then $M \cap N$ is a union of codimension-1 faces of $\overline{\mathcal{C}}$, each of which defines an edge in $\overline{\mathcal{E}}$, so $M-N$ is in the edge set of $\mathcal{C}^{\prime}$ as a coarsening of $\mathcal{C}$. If not, then $M \cap N$ is a union of codimension-1 faces of $\overline{\mathcal{C}}$ none of which defines an edge in $\overline{\mathcal{E}}$, so $M-N$ is not in the edge set of $\mathcal{C}^{\prime}$ as a coarsening of $\mathcal{C}$. We have proved the general case of Theorem 1.1 .

We conclude the section by adapting the above arguments to prove a special case of Tietze's convexity theorem. This will be generalized to oriented matroids as Theorem [5.5.

Theorem 4.3. Let $\mathcal{M}$ be a finite set of $n$-dimensional polyhedra in $\mathbb{R}^{n}$. Suppose:

(i) The interior of $\operatorname{Supp}(\mathcal{M})$ is path-connected.

(ii) For every $x$ in the boundary of $\operatorname{Supp}(\mathcal{M})$, there exists a closed half-space $H^{+}$bounded by a hyperplane $H$ such that $x \in H$ and $\bigcup_{x \in M \in \mathcal{M}} M \subseteq H^{+}$.

Then $\operatorname{Supp}(\mathcal{M})$ is convex.

Proof. Let $\mathcal{A}$ be the set of hyperplanes that contain codimension-1 faces of polyhedra in $\mathcal{M}$. Let $\overline{\mathcal{M}}$ be the set of regions of $\mathcal{A}$ contained in $\operatorname{Supp}(\mathcal{M})$. Let $\overline{\mathcal{E}}$ be the set of edges $Q-R$ in $\mathcal{G}(\mathcal{A})$ such that $Q$ and $R$ are both in $\overline{\mathcal{M}}$. Let $U$ be the union of all faces of codimension at least 2 of polyhedra of $\overline{\mathcal{M}}$. Since the interior of $\operatorname{Supp}(\mathcal{M})=\operatorname{Supp}(\overline{\mathcal{M}})$ is path-connected and full-dimensional, for any two polyhedra $M$ and $N$ in $\overline{\mathcal{M}}$, there exists a continuous path in $\operatorname{int}(\operatorname{Supp}(\overline{\mathcal{M}})) \backslash U$ from $\operatorname{int}(M)$ to $\operatorname{int}(N)$. This implies that $\overline{\mathcal{M}}$ is a connected component of $\overline{\mathcal{E}}$.

Let $\mathcal{P}$ be a $2 k$-gon in $\mathcal{G}(\mathcal{A})$ defined by a codimension-2 face $F$ of $\mathcal{C}(\mathcal{A})$. Suppose that $k$ consecutive edges of $\mathcal{P}$ are in $\overline{\mathcal{E}}$ but that not all of the edges of $\mathcal{P}$ are in $\overline{\mathcal{E}}$. Then any point $x$ in the relative interior of $F$ provides a violation of hypothesis (ii). Thus $\overline{\mathcal{E}}$ has the weak polygon property, so $\overline{\mathcal{E}}$ defines a pre-complex $\mathcal{C}^{\prime}$ by Lemma 4.1 (with $\mathcal{C}=\mathcal{C}(\mathcal{A})$ ). Since $\overline{\mathcal{M}}$ is a connected component of $\overline{\mathcal{E}}$, one of the full-dimensional polyhedra in $\mathcal{C}^{\prime}$ is $\operatorname{Supp}(\overline{\mathcal{M}})=\operatorname{Supp}(\mathcal{M})$.

\section{Polyhedral Complexes in oriented matroids}

In this section, we extend our results to the context of oriented matroids. We base our approach to oriented matroids on [1, Chapter 4].

Let $\mathcal{L} \subseteq\{+,-, 0\}^{E}$ be the set of covectors of an oriented matroid with no loops, over a finite ground set $E$. The symbol $\mathcal{L}$ will also denote the corresponding ("big") face lattice. A closed half-space in $\mathcal{L}$ is a set $H_{e}^{+}$consisting of all covectors in $\mathcal{L}$ having component + or 0 in position $e$, or a set $H_{e}^{-}$consisting of all covectors having component - or 0 in position $e$. (In [1, Chapter 4.2], a different notion of half-spaces appears, but translating between the two conventions is easy.) A hyperplane in $\mathcal{L}$ is a set $H_{e}=H_{e}^{+} \cap H_{e}^{-}$. A polyhedron $P$ in $\mathcal{L}$ is an intersection of closed half-spaces. The rank of a polyhedron $P$ is the rank of the maximal covectors in $P$. An exposed face of $P$ is a subset of $P$ of the form $P \cap H_{e}$ for any $e \in E$ with $P \subseteq H_{e}^{+}$or $P \subseteq H_{e}^{-}$. A face of $P$ is any intersection of exposed faces, 
including the empty intersection, which is interpreted as $P$. A set $U$ of covectors is closed if it is an order ideal in $\mathcal{L}$. The boundary of a closed set $U$ of covectors is the set of covectors $X$ in $U$ such that the principal order filter of $\mathcal{L}$ generated by $X$ is not contained in $U$. The interior of $U$ is $U$ minus the boundary of $U$. A set of covectors is connected if it induces a connected subgraph of the Hasse diagram of $\mathcal{L}$.

The definition of a polyhedral complex in $\mathcal{L}$ and terminology for complexes is copied verbatim from Section 1 , with $\mathbb{R}^{n}$ replaced by $\mathcal{L}$.

Theorem 5.1. Fix $k \geq-1$ and let $\mathcal{M}$ be a finite collection of polyhedra in an oriented matroid $\mathcal{L}$, each of rank greater than $k$. Suppose:

(i) For each $X \in \operatorname{Supp}(\mathcal{M})$, the set $\left\{Y \in \operatorname{Supp}(\mathcal{M}) \backslash \bigcap_{k}(\mathcal{M}): Y \geq X\right\}$ is connected. (Here $\bigcap_{k}(\mathcal{M})$ is defined as in Section 1].)

(ii) $M \cap N$ is a face of $M$ and of $N$ for all $M, N \in \mathcal{M}$ with $\operatorname{rank}(M \cap N)>k$.

Then the collection of all polyhedra in $\mathcal{M}$ and their faces is a polyhedral complex.

Proof. The oriented matroid version of Lemma 2.1 holds by a proof that is the same, except for a modification of the argument that $F \cap N$ is a face of $M \cap N$. For each exposed face $F^{\prime}$ of $M$ containing $F$, we argue as in the proof of Lemma 2.1 that $F^{\prime} \cap N$ is an exposed face of $M \cap N$. Since $F$ is the intersection of all exposed faces of $M$ containing it, we conclude that $F \cap N$ is an intersection of exposed faces of $M \cap N$, or in other words that $F \cap N$ is a face of $M \cap N$.

Let $M$ and $N$ be distinct polyhedra in $\mathcal{M}$, let $F$ be the polyhedron $M \cap N$, and let $d$ be the rank of $F$. Let $X$ be a rank- $d$ covector in $F$. Then the covectors in $\operatorname{Supp}(\mathcal{M}) \backslash \bigcap_{k}(\mathcal{M})$ that are above $X$ in the lattice $\mathcal{L}$ form a connected set. Let $Y$ be a covector of full rank in $M$ and $Z$ a covector of full rank in $N$. Then there is a path $Y=X_{0}, X_{1}, \ldots, X_{j}=Y$ in $\operatorname{Supp}(\mathcal{M}) \backslash \bigcap_{k}(\mathcal{M})$ consisting of covectors above $X$. Let $M_{0}=M$ and $M_{j}=N$ and for each $i=1, \ldots, j-1$, let $M_{i}$ be any polyhedron in $\mathcal{M}$ with $X_{i} \in M_{i}$. The result is a sequence $M=M_{0}, \ldots, M_{j}=N$ of polyhedra in $\mathcal{M}$, all containing $X$, such that, for each $i=1, \ldots, j$, the intersection $M_{i-1} \cap M_{i}$ is of rank greater than $k$. As in the proof of Theorem 1.2, we see that $F^{\prime}=M_{0} \cap \cdots \cap M_{j}$ is a face of $N$. By construction, $F^{\prime}$ contains $X$ and so has rank at least $d$. Since $F^{\prime} \subseteq F$, it has rank exactly $d$.

Let $G=N \cap H$ be any exposed face of $N$ containing $F^{\prime}$. Then $F^{\prime} \subseteq H$. Since $F$ and $F^{\prime}$ are rank- $d$ polyhedra with $F^{\prime} \subseteq F$, we have $F \subseteq H$ and so $F \subseteq G$. Thus $F \subseteq F^{\prime}$, so $F=F^{\prime}$ is a face of $N$. By symmetry, $F$ is a face of $M$.

If the rank of $\mathcal{L}$ is $n$, then $\{X \in \mathcal{L}: \operatorname{rank}(X) \in\{n-1, n\}\}$ is connected by [1, Proposition 4.2.3]. The following proposition is [1, Proposition 4.2.6].

Proposition 5.2. Let $P$ be an order ideal in $\mathcal{L}$ generated by a set of covectors of full rank $n$. Then $P$ is a polyhedron if and only if the following condition holds: If $Y$ and $Z$ are maximal in $P$ and $X$ is on a shortest path from $X$ to $Y$ in $\{X \in \mathcal{L}: \operatorname{rank}(X) \in\{n-1, n\}\}$, then $X \in P$.

Paths and path convexity in $\mathcal{L}$ are defined as in the realizable case. Replacing Theorem 3.4 with [2, Theorem 2.4] and establishing, by the same proof, the analog of Lemma 3.3. we obtain the following theorem. (Cf. [1, Proposition 4.4.6].)

Theorem 5.3. Every oriented matroid is path convex. 
Let $F$ be a corank-2 face of a polyhedral complex $\mathcal{C}$ in $\mathcal{L}$. Define $\operatorname{Perp}(F)$ to be the rank-2 oriented matroid obtained by the deletion from $\mathcal{L}$ of all $e \in E$ such that $H_{e}$ does not contain $F$. Since every rank-2 oriented matroid is realizable, we can think of $\operatorname{Perp}(F)$ as a fan. For each $M \in \mathcal{M}$ with $F \subset M$, define a cone in $\operatorname{Perp}(F)$ as the set of covectors in $\operatorname{Perp}(F)$ which arise by deletion from covectors in $M$. The fan $\left.\mathcal{C}\right|_{F}$ is the collection of all such cones. The ridge property on a set $\mathcal{E}$ of edges in the adjacency graph $\mathcal{G}$ is now defined exactly as in the realizable case.

Theorem 5.4. Let $\mathcal{C}$ be a pure, full-rank, locally strongly connected polyhedral complex in an oriented matroid $\mathcal{L}$. Then a subset $\mathcal{E}$ of the edges of the adjacency graph $\mathcal{G}$ is the edge set of a complex coarsening $\mathcal{C}$ if and only if $\mathcal{E}$ has the ridge property.

The proof of Theorem 5.4 runs along the same lines as the proof of Theorem 1.1 . First, the oriented-matroid analog of Lemma 4.1 holds by essentially the same proof, but instead of considering a line segment between points in $M$, we appeal to Proposition 5.2. The analog of Lemma 4.2 also holds by the same proof, except that instead of using a line segment $\overline{x x^{\prime}}$ to construct a sequence of corank-1 covectors contained in $F$, we appeal to Proposition 5.2, applied to the restriction of $\mathcal{L}$ to the hyperplane containing $F$.

Let $\mathcal{C}$ be a pure, full-rank, locally strongly connected polyhedral complex in $\mathcal{L}$. Given a covector $X$, the notation $\bar{X}$ will refer to the polyhedron which is the principal order ideal below $X$ in $\mathcal{L}$. Thus $\bar{X}$ should be thought of as the closure of $X$, while $X$ should be thought of as the relative interior of $\bar{X}$. Let $\mathcal{C}(\mathcal{L})$ be the polyhedral complex in $\mathcal{L}$ consisting of all polyhedra $\bar{X}$ such that $X$ is a covector in $\mathcal{L}$. Let $\overline{\mathcal{C}}$ be the subcomplex of $\mathcal{C}(\mathcal{L})$ consisting of faces contained in $\operatorname{Supp}(\mathcal{C})$. Then $\mathcal{C}$ coarsens $\overline{\mathcal{C}}$. As in the realizable case, we begin with a set $\mathcal{E}$ of edges in the adjacency graph of $\mathcal{C}$, with the ridge property, and construct a set $\overline{\mathcal{E}}$ of edges of the adjacency graph of $\overline{\mathcal{C}}$. We then argue, along the same lines, that $\overline{\mathcal{E}}$ has the ridge property. Using the oriented-matroid analogs of Lemmas 4.1 and 4.2 as well as Proposition 5.2, we verify the hypotheses of Theorem 5.1 and conclude that $\overline{\mathcal{E}}$ is the edge set of a complex $\mathcal{C}^{\prime}$ coarsening $\overline{\mathcal{C}}$. The argument that $\mathcal{E}$ is the edge set of $\mathcal{C}^{\prime}$ as a coarsening of $\mathcal{C}$ extends to the oriented matroid case, and this completes the proof of Theorem 5.4.

Echoing the proof of Theorem 4.3, with $\mathcal{C}(\mathcal{A})$ replaced by $\mathcal{C}(\mathcal{L})$, we obtain an oriented matroid version of Tietze's convexity theorem.

Theorem 5.5. Let $\mathcal{M}$ be a finite set of polyhedra of full rank in $\mathcal{L}$. Suppose:

(i) The interior of $\operatorname{Supp}(\mathcal{M})$ is connected.

(ii) For all $X$ in the boundary of $\operatorname{Supp}(\mathcal{M})$, there exists a closed half-space $H^{+}$ bounded by a hyperplane $H$ such that $X \in H$ and $\bigcup_{x \in M \in \mathcal{M}} M \subseteq H^{+}$.

Then $\operatorname{Supp}(\mathcal{M})$ is a polyhedron in $\mathcal{L}$.

Remark 5.6. When $\mathcal{L}$ is realizable, polyhedral complexes in $\mathcal{L}$, as defined above, are fans rather than polyhedral complexes. One can similarly define polyhedral complexes and prove all of the results of this section in the context of affine oriented matroids as in [1, Section 4.5]. As usual, the decision to pass from the linear to the affine or vice versa is a matter of convenience, not a matter of mathematical substance. 


\section{ACKNOWLEDGMENTS}

The author gratefully acknowledges the contributions of several colleagues: Bernd Sturmfels made the author aware of the problem of generalizing [4, Theorem 9] from the symmetric group to arbitrary finite Coxeter groups and contributed helpful comments. Ezra Miller suggested that the argument for Theorem 1.1 should imply some local condition for convexity (see Theorem 4.3). Sergei Ivanov answered the author's question on mathoverflow.net, making the author aware of Tietze's convexity theorem and giving a reference. Isabella Novik, Igor Pak, Vic Reiner, and an anonymous referee contributed helpful comments and references. In an earlier version of this paper, Theorems 1.1 and 5.4 were proved under the stronger hypothesis that $\mathcal{C}$ has convex support. The referee pointed out that the argument goes through, with only trivial modifications, for $\mathcal{C}$ pure, full-dimensional, and locally strongly connected.

\section{REFERENCES}

[1] A. Björner, M. Las Vergnas, B. Sturmfels, N. White and G. Ziegler, Oriented matroids (Second edition), Encyclopedia of Mathematics and its Applications, 46, Cambridge Univ. Press, 1999. MR 1744046 (2000j:52016)

[2] R. Cordovil and M. L. Moreira, A homotopy theorem on oriented matroids. Discrete Math. 111 (1993), no. 1-3, 131-136. MR1210090 (94d:52016)

[3] P. Deligne, Les immeubles des groupes de tresses généralisés. Invent. Math. 17 (1972), 273302. MR0422673 (54:10659)

[4] J. Morton, L. Pachter, A. Shiu, B. Sturmfels and O. Wienand, Convex rank tests and semigraphoids. SIAM J. Discrete Math. 23 (2009), no. 3, 1117-1134. MR 2538642 (2011b:62126)

[5] N. Reading, Lattice congruences, fans and Hopf algebras. J. Combin. Theory Ser. A 110 (2005), no. 2, 237-273. MR2142177 (2006b:20054)

[6] M. Salvetti, Topology of the complement of real hyperplanes in $C^{N}$. Invent. Math. 88 (1987), no. 3, 603-618. MR884802 (88k:32038)

[7] H. Tietze, Bemerkungen über konvexe und nicht-konvexe Figuren. J. Reine Angew. Math. 160 (1929), 67-69.

[8] J. Tits, Le problème des mots dans les groupes de Coxeter. 1969 Symposia Mathematica (INDAM, Rome, 1967/68), Vol. 1, 175-185. Academic Press, London. MR0254129 (40:7339)

[9] F. A. Valentine, Convex sets. McGraw-Hill Book Co., New York-Toronto-London, 1964. MR0170264(30:503)

[10] G. Ziegler, Lectures on polytopes. Graduate Texts in Mathematics, 152. Springer-Verlag, New York, 1995. MR1311028(96a:52011)

Department of Mathematics, North Carolina State University, Raleigh, North CaroLINA 27695 